\title{
Associations Between Antibodies Against the Endothelial Cell and T. gondii; Cytomegalovirus in Serum of Children with Cochlear Implant Surgery
}

\section{Samileh Noorbakhsh ${ }^{1}$, Mohammad Farhadi ${ }^{2}$, Ahamad Daneshi ${ }^{3}$, Azardokht Tabatabaei ${ }^{4}$, Emam H jomeh ${ }^{5}$ and Yaser Ghavami ${ }^{6}$}

${ }^{1}$ Professor of Pediatric Infectious Diseases, Research Center of Pediatric Infectious Diseases, Tehran University of Medical Sciences, Tehran, Iran ${ }^{2}$ Professor of ENT, ENT -Head \& Neck Surgery Research Center, Tehran University of Medical Sciences, Tehran, Iran ${ }^{3}$ Professor of ENT; ENT -Head \& Neck Surgery Research Center, Tehran University of Medical Sciences, Tehran, Iran ${ }^{4}$ Microbiologist, Research Center of Pediatric Infectious Diseases, Tehran University of Medical Sciences, Iran

${ }^{5}$ Audiologist, ENT -Head \& Neck Surgery Research Center, Tehran University of Medical Sciences, Iran

${ }^{6}$ General Practitioner, ENT -Head \& Neck Surgery Research Center, Tehran University of Medical Sciences, Tehran, Iran

\begin{abstract}
Background: Cytomegalovirus (CMV) and T. gondii are two common causes of SNHL (Sensorineural hearing loss) in Iranian children. Immune-mediated vascular damage induced by endothelial cell antibodies may have a prominent role in sensorineural hearing loss.
\end{abstract}

Objectives: To determine the serum CMV and T. gondii antibodies against endothelial cell in children with profound SNHL and cochlear implant surgery.

Materials \& Methods: A cross sectional study was performed on 76 cases with severe SNHL (mean age $32 \pm$ 30.6 months) at cochlear implant ward of Rasoul hospital, Tehran Iran (2008-2010). The titers of antibodies against endothelial cell (Indirect immunofluorescence assay); were determined in sera of 66 cases. Specific antibodies (IgG \& IgM) against $T$. gondi, CMV (Enzyme linked immunosorbent assay) determined in Idiopathic SNHL cases.

Results: Idiopathic type of SNHL was diagnosed in $28.8 \%(19 / 66)$ of younger cases (mean age=20 months; $P V=0.05)$. Positive AECAs was detected in 14.4\% (11/76) of cases (with mean age 50 vs. 32 months in cases with negative test; $P$ value $=0.047$ ). Positive AECAs had not significant differences between Idiopathic and Non idiopathic type of SNHL [10.5\% vs. 9\%; PV=0.1]. Positive AECAs were more frequent in cases with known postnatal infections (e.g. mumps, meningitis, chicken pox, etc.) in comparison with non-infection cases (P-value $=0.05)$. Positive T. gondii -IgM (recent infection) was found in $8 / 19$ (\%42); 1 case also had positive T. gondii -lgG. Positive CMV-IgM \& IgG were determined in $10 / 19(\% 52) ; 17 / 19(\% 89)$ respectively. A meaningful correlation was observed between positive AECAs and those infections (Toxo, CMV) in Idiopathic (and unclassified) SNHL cases.

Conclusion: Idiopathic type of SNHL with a poor outcome is common $(28.8 \%)$ in children with cochlear implant surgery Positive AECAs cases had not differences between 2 type of SNHL but were more frequent in older cases with known postnatal infections (meningitis, mumps, chicken pox etc.). A good correlation $(p=0.05)$ between the positive AECAs and known infections determined in studied cases. In contrast, those younger cases ( $<3$ years) with positive AECAs had recent CMV /or T. Gondii (Positive IgM) infections (23\%; 17.7\%) without known congenital or acquired type of infections. At least in our country, in younger SNHL cases ( $<2$ year old) due to confirmed recent CMV or T. Gondii infections, application of specific drugs are preferred. Immunosuppressive therapy is only recommended in older cases (>2 year old) with Idiopathic SNHL and concomitant positive AECAs.

Keywords: Sensory neural hearing loss (SNHL); Cochlear implant; Anti-endothelial cell antibodies (AECAs); CMV; T. Gondii

\section{Introduction}

The incidence of unilateral hearing loss in children was approximately $0.1 \%$. In $7.5 \%$ of cases unilateral deafness were diagnosed accidentally, most often between the 7 th and the $10^{\text {th }}$ decades of age [1] The etiology of most of these cases remains unknown. Neither children nor their parents can precisely determine the time of its appearance, especially when it is not accompanied by other symptoms, such as dizziness or tinnitus [2]. Infants hospitalized in Neonatal Intensive Care Units (NICU) are at higher risk for SNHL [3,4]. The overall incidence of inner ear abnormalities in children with SNHL evaluated by MRI is $40 \%$. Children with unilateral hearing loss have a greater percentage of inner ear anomalies than children with bilateral SNHL [5]. Asymptomatic or symptomatic course of mumps, CMV, T. gondii and measles should also be taken into consideration as causes of SNHL in children. Autoimmune hearing loss as a plausible explanation for a certain percentage of the group is categorized as Idiopathic type. SNHL in children can be caused by autoimmune disorders localized to the inner ear or secondary to systemic immune diseases. Many studies established the non-specific auto-antibodies vs. the inner ear, such as anti endothelial cell antibodies (AECAs). AECAs have a prognostic

*Corresponding author: Samileh Noorbakhsh, Professor of Pediatric Infectious Diseases, Research Center of Pediatric Infectious Diseases, 4th floor Hazrat Rasul Hospital, Niayesh Street, Satarkhan Avenue, Tehran, 14455 Islamic Republic of Iran, Tel: 098-21-66525328; Fax: 098-21-66516049; E-mail: Samileh_noorbakhsh@yahoo.com

Received February 26, 2013; Accepted March 22, 2013; Published March 26 2013

Citation: Noorbakhsh S, Farhadi M, Daneshi A, Tabatabaei A, jomeh EH, et al (2013) Associations Between Antibodies Against the Endothelial Cell and T. gondii; Cytomegalovirus in Serum of Children with Cochlear Implant Surgery. J AIDS Clin Res 4: 197. doi:10.4172/2155-6113.1000197

Copyright: (c) 2013 Noorbakhsh S, et al. This is an open-access article distributed under the terms of the Creative Commons Attribution License, which permits unrestricted use, distribution, and reproduction in any medium, provided the original author and source are credited. 
factor for these diseases and can be considered a useful clinical tool for differentiating patients with idiopathic hearing loss [6-8].

Cadoni et al. investigated the presence of AECAs and its role in causing damage to the stria vascularis in immune-mediated sensorineural deafness [9]. The appearance of endothelial cell antibody is related to the poor outcome of hearing loss [10-16].

Detection of the serum AECAs can be helpful in selection of particular patients with SNHL for specific immunosuppressive treatments [17-22].

Congenital CMV is the most common cause of congenital infections in the world [23-25]. In last decade, role of CMV and T. Gondii infections in children with SNHL had been proved in our center [26,27].

Main goal of this study was to determine associations between serum AECAs and antibodies against CMV and T. Gondii in children with profound SNHL (cochlear implant surgery) in our center.

\section{Methods and Materials}

This case - control study was performed in cochlear implant center of Rasul Akram Hospital in Tehran from 2008 to 2010.

Cases consisted of 75 children with Severe SNHL ( $<95 \mathrm{db})$ which were candidate for cochlear implant surgery. This study was approved by the Ethical Committee in Research Center of ENT, Head and Neck Surgery \& Research Center of Pediatric Infectious Diseases in Tehran University of Medical Sciences. Initially, a questionnaire was completed by an authorized physician for each case and control.

Audiologic screening (Auditory Brainstem Response, Evoked Ottoacoustic Emissions and Pure Tone Audiometry) appropriate for age was used in all cases. Diagnostic parameters for SNHL were based on AAO (American academy of Otolaryngology criteria). Idiopathic and Non idiopathic type of SNHL were diagnosed in all cases.

Blood samples $(2 \mathrm{ml})$ were centrifuged and transferred to our research laboratory. All sera were kept frozen at $-80^{\circ} \mathrm{C}$ until usage. AECAs (IgG) searched in serum by Indirect Fluorescent Antibody Test (KMI diagnostics, US); ELISA assay was used for searching the specific IgM and IgG antibodies against CMV, T. Gondii (Bio chem Immuno Systems, Italy). The results were calculated qualitatively as suggested by the manufacturer. In order to minimize the false-positive interferences with anti-endothelial cell antibodies (AECAs), Rheumatoid factors (RFs) and antinuclear antibodies (ANAs) titers followed in serum samples in the cases. All positive individuals for RFs \& ANAs ( 5 cases and 3 controls) were excluded.

\section{Statistical analysis}

The Student t-test was used to determine significant differences in means for all continuous variables. Chi-square values (CI: 95\%; $\mathrm{P}<0.05$ ) were calculated for all categorical variables. $\mathrm{P}$ value less than 0.05 was considered significant. All analyses were conducted using SPSS version 11.5 .

\section{Results}

The age range in SNHL cases $(\mathrm{n}=75)$ were between 3 to 168 months (mean $=33.6 \pm 38.6$ months). 50\% (37/74) were male and 50\% (37/74) were female (missing $=2)$.

Positive AECAs were detected in 14.5\% (11/75) of SNHL cases; AECAs were seen in cases aged $>5$ years in comparison with cases $<5$ years old $(\mathrm{PV}=0.047)$. The mean age of children with positive AECAs was higher (50 vs. 32 months $=0.05$ ). Positive AECAs had no differences between Idiopathic (and unclassified); and Non Idiopathic type of SNHL [10.5\% (2/21) vs. 9\% (4/47) PV=0.1]. Positive AECAs were more frequent in cases with known postnatal infections infections (e.g.: mumps, meningitis, chicken pox, etc.) in comparison with noninfections cases $(\mathrm{P}$-value $=0.05)$ (Table 1$)$.

Idiopathic type of SNHL was diagnosed in $28.8 \%(19 / 66)$ of cases. Non Idiopathic SNHL (e.g. familial, kernicterus, prematurity, hypoxic ischemic, infections etc.) was diagnosed in $71.2 \%$ (47/66); missing (unclassified) reported in 8 cases. Mean age of Idiopathic SNHL cases was lower than Non Idiopathic type (20 vs. 45 months; $P=0.05$ ).Positive AECAs had no differences between Idiopathic and Non Idiopathic type of SNHL [10.5\% (2/19) vs. $9 \%(4 / 47), \mathrm{p}=0.1]$

Searching the sera for infections had done for all idiopathic cases. Positive T. Gondii -IgM (recent infection) was found in 8/19 (\%42); 1 case also had positive T. Gondii -IgG. Positive CMV-IgM and IgG were determined in 10/19 (\%52); 17/19 (\%89) respectively. A meaningful correlation was observed between positive AECAs and those infections (Toxo, CMV) in Idiopathic (and unclassified) SNHL cases.

\section{Discussion}

In present study, 30\% (19/76) of studied children were diagnosed as Idiopathic type of SNHL which is very close to $38.7 \%$ reported by other authors [1-4]. Idiopathic SNHL cases were younger than Nonidiopathic type ( 20 vs. 45 months).

On the other hand, Results of this study is far from Cadonni et al. [14] study in adults; they reported the higher positive AECAs (54\%) in SNHL cases than control group $(\mathrm{P}=0004)$.

Positive AECAs were not different between Idiopathic and Non idiopathic type of SNHL [10\% vs. $9 \%, \mathrm{P}=0.1)$. Cases with positive AECAs were older than 5 years (Fisher's Exact Test=0.047).

Although positive AECAs were not significantly different between 2 types of SNHLs, But definite postnatal infectious causes (meningitis, mumps, chicken pox, etc.) and recent T. gondii and CMV infection were observed in Non-Idiopathic type of SNHL with positive AECAs (>5year old).

\begin{tabular}{|c|c|c|c|}
\hline $\begin{array}{l}\text { SNHL cases } \\
\text { (Mean age }=33.6 \text { months) } \\
\text { Missed (Unclassified=8) }\end{array}$ & $\begin{array}{l}\text { Idiopathic SNHL }(28 \%) \\
\text { (Mean age }=20 \text { months) }\end{array}$ & $\begin{array}{l}\text { Non Idiopathic SNHL (\%71.2) } \\
\quad(\text { Mean age }=45 \text { months })\end{array}$ & $P$ value $=0.05$ \\
\hline $\begin{array}{l}\text { Positive AECAs } \\
11 / 75(\% 14.4)\end{array}$ & $2 / 19(\% 10.5)$ & $4 / 47(\% 9)$ & $P$ value $=0.1$ \\
\hline $\begin{array}{l}\text { Positive } T \text {. Gondii IgM } \\
8 / 45(\% 17.7)\end{array}$ & $\begin{array}{c}\text { Positive T. Gondii IgM } \\
8 / 19(\% 42)\end{array}$ & ----- & --- \\
\hline $\begin{array}{l}\text { Positive CMV IgM } \\
10 / 45(\% 23)\end{array}$ & $\begin{array}{c}\text { Positive CMV IgM } \\
10 / 19(\% 52)\end{array}$ & ----- & ------ \\
\hline
\end{tabular}

Table 1: Comparing the AECAs and T. Gondii /CMV antibodies between SNHL cases. 
Citation: Noorbakhsh S, Farhadi M, Daneshi A, Tabatabaei A, jomeh EH, et al. (2013) Associations Between Antibodies Against the Endothelial Cell and T. gondii; Cytomegalovirus in Serum of Children with Cochlear Implant Surgery. J AIDS Clin Res 4: 197. doi:10.4172/2155-6113.1000197

Page 3 of 4

Positive T. gondii -IgM (recent infection) was found in $17.7 \%$ of cases $(8 / 45) ; 1$ case $(2.2 \%)$ had positive T. gondii $-\mathrm{IgG}$ (previous infection). Positive CMV-IgM \&IgG were 11/45 (23\%), 23/45 (51\%) respectively.

Post or prenatal (usually indolent) T.Gondii /CMV infections were frequent in present study. Recent CMV infection (positive IgM) in $23 \%$ and recent T.Gondii infection in $17.7 \%$ of idiopathic (and unclassified) type of SNHL cases. These results are very close to previous serologic study in our center [26-29]. Recent CMV and T. Gondii infections (positive IgM) in SNHL cases (mean age $=35$ months) were $34.6 \%$ and $11.5 \%$, respectively. But other infections (Mumps, Rubella, Herpes type-1) were rare [29]. The previous study in all type of SNHL in our center had similar results [27]. Recent T.Gondii infection (IgM) detected in none of normal controls in compare with $12 \%$ of SNHL cases $(\mathrm{P}$ value $=0.00)$, but previous immunity $(\mathrm{IgG})$ against T.Gondii infection was significantly higher in the control healthy group ( $48 \%$ vs $21 \%$; P value $<0.001$ ) [27].

As previous studies, other infections might have an etiologic role in deafness in Iran [27-29]. Specific drugs for confirmed recent CMV infections in SNHL children ( $<2$ years) are recommended [23-26].

In addition to postnatal infectious diseases (mumps; chicken pox; severe sepsis, otitis media, etc.) which were observed in cases, an excellent correlation was seen between positive AECAs and positive antibodies against $T$. gondii/CMV in Idiopathic type of SNHL (mean age: 22 months). T. gondii infection was defined as the most common infection with positive AECAs. Congenital (or acquired) Toxo/CMV infections might have role in younger cases with Idiopathic type of SNHL ( $<2$ years).

Role of CMV in pathogenesis of SNHL in children have been reported in many countries as well as Iran. Correlation between proven infection and positive AECAs does not mean the cause and effect in studied cases.

Positive AECAs in older Idiopathic SNHL cases ( $>5$ year) could define the clinical associations of AECAs with immune-mediated inner-ear disorders. Probably AECAs after infancy period might play a prominent role in causing damage to the stria vascularis in immunemediated SNHL. Multiple potential mechanisms can result in immunemediated inner ear disease in children. Except one study in children, other previous studies had been done in adults [17]. The association of AECAs with endothelial injury in the course of these diseases prompted us to develop assays for these antibodies in clinical practice. Prognostic factors for Idiopathic SNHL in adults were reported by many authors [10-14].

Production of serum AECAS would be as a marker of disease activity. Cvorović et al. reported the appearance of AECAs is related to the poor outcome and absent recovery of hearing loss in adults [10].

Many authors offered the treatment of SNHL in adults with systemic or intra tympanic steroids [15-20].

Westerlaken et al. and Tucci et al. treated the Idiopathic SNHL cases with combination of steroids and antiviral drugs $[21,22]$.

Due to poor outcome of Idiopathic SNHL in children, we recommend to search not only the serum AECAs, but also specific antibodies against indolent CMV/T.Gondii infections in the cases. Idiopathic type of SNHL observed in lower age $(<2$ years old $)$ might have congenital (or acquired) Toxo/CMV infections which can induce progressive hearing loss. Fortunately both infections are treatable by specific oral drugs. Decisions for immunosuppressive therapy in older children need a randomized clinical trial in future.

\section{Strength of the study}

Correlation between indolent CMV/T.Gondii infections and AECAs in children with Idiopathic type of SNHL has not been studied up to now. Most previous studies done in adults but not in children especially in Idiopathic type of SNHL.

\section{Limitations of the study}

Small population especially in younger age ( $<2$ year old $)$ in present study is an important limitation. Other infectious agents (influenza, measles, mumps, varicella etc.) were not studied in our cases.

\section{Conclusion}

Idiopathic type of SNHL with a poor outcome is common $(28.8 \%)$ in children with cochlear implant surgery Positive AECAs cases had not differences between 2 type of SNHL but were more frequent in older cases with known postnatal infections (meningitis, mumps, chicken pox etc.). A good correlation $(\mathrm{p}=0.05)$ between the positive AECAs and known infections determined in studied cases. In contrast, those younger cases ( $<3$ years) with positive AECAs had recent CMV /or T. Gondi (Positive IgM) infections (23\%; 17.7\%) without known congenital or acquired type of infections. At least in our country, in younger SNHL cases $(<2$ year old) due to confirmed recent CMV or T. Gondi infections, application of specific drugs are preferred. Immunosuppressive therapy is only recommended in older cases $(>2$ year old) with Idiopathic SNHL and concomitant positive AECAs.

\section{Acknowledgments}

This study was supported by the ENT, Head and Neck \& Surgery, and Research Center of Pediatric Infectious Diseases in Tehran University of Medical Sciences.

\section{References}

1. Olusanya BO, Okolo AA (2006) Adverse perinatal conditions in hearingimpaired children in a developing country. Paediatr Perinat Epidemiol 20: 366371.

2. Walch C, Anderhuber W, Köle W, Berghold A (2000) Bilateral sensorineura hearing disorders in children: etiology of deafness and evaluation of hearing tests. Int J Pediatr Otorhinolaryngol 53: 31-38.

3. Kountakis SE, Skoulas I, Phillips D, Chang CY (2002) Risk factors for hearing loss in neonates: a prospective study. Am J Otolaryngol 23: 133-137.

4. Martínez-Cruz CF, Poblano A, Fernández-Carrocera LA (2008) Risk factors associated with sensorineural hearing loss in infants at the neonatal intensive care unit: 15-year experience at the National Institute of Perinatology (Mexico City). Arch Med Res 39: 686-694.

5. Cadoni G, Fetoni AR, Agostino S, De Santis A, Vulpiani P, et al. (2001) [Role of endothelial cell autoantibodies in the pathogenesis of sudden hear loss]. Acta Otorhinolaryngol Ital 21: 138-143.

6. Vasama JP, Linthicum FH Jr (2000) Idiopathic sudden sensorineural hearing loss: temporal bone histopathologic study. Ann Otol Rhinol Laryngol 109: 527 532.

7. Merchant SN, Adams JC, Nadol JB Jr (2005) Pathology and pathophysiology of idiopathic sudden sensorineural hearing loss. Otol Neurotol 26: 151-160.

8. Cadoni G, Agostino S, Manna R, De Santis A, Fetoni AR, et al. (2003) Clinical associations of serum antiendothelial cell antibodies in patients with sudden sensorineural hearing loss. Laryngoscope 113: 797-801.

9. Cvoroviä $\ddagger$ L, Deric D, Probst R, Hegemann S (2008) Prognostic model for predicting hearing recovery in idiopathic sudden sensorineural hearing loss. Otol Neurotol 29: 464-469.

10. Xenellis J, Karapatsas I, Papadimitriou N, Nikolopoulos T, Maragoudakis P, et 
Citation: Noorbakhsh S, Farhadi M, Daneshi A, Tabatabaei A, jomeh EH, et al. (2013) Associations Between Antibodies Against the Endothelial Cell and T. gondii; Cytomegalovirus in Serum of Children with Cochlear Implant Surgery. J AIDS Clin Res 4: 197. doi:10.4172/2155-6113.1000197

al. (2006) Idiopathic sudden sensorineural hearing loss: prognostic factors. J Laryngol Otol 120: 718-724.

11. Solares CA, Hughes GB, Tuohy VK (2003) Autoimmune sensorineural hearing loss: an immunologic perspective. J Neuroimmunol 138: 1-7.

12. Naumann A, Hempel JM, Schorn K (2001) [Detection of humoral immune response to inner ear proteins in patients with sensorineural hearing loss]. Laryngorhinootologie 80: 237-244.

13. Ceylan A, Celenk F, KemaloÄŸlu YK, Bayazit YA, GÃ Tksu N, et al. (2007) Impact of prognostic factors on recovery from sudden hearing loss. J Laryngol Otol 121: 1035-1040.

14. Agrup C, Luxon LM (2006) Immune-mediated inner-ear disorders in neurootology. Curr Opin Neurol 19: 26-32.

15. Chen YS, Emmerling O, Ilgner J, Westhofen M (2005) Idiopathic sudden sensorineural hearing loss in children. Int J Pediatr Otorhinolaryngol 69: 817821.

16. Herr BD, Marzo SJ (2005) Intratympanic steroid perfusion for refractory sudden sensorineural hearing loss. Otolaryngol Head Neck Surg 132: 527-531.

17. Gouveris H, Selivanova O, Mann W (2005) Intratympanic dexamethasone with hyaluronic acid in the treatment of idiopathic sudden sensorineural hearing loss after failure of intravenous steroid and vasoactive therapy. Eur Arch Otorhinolaryngol 262: 131-134.

18. Plontke S, Löwenheim H, Preyer S, Leins P, Dietz K, et al. (2005) Outcomes research analysis of continuous intratympanic glucocorticoid delivery in patients with acute severe to profound hearing loss: basis for planning randomized controlled trials. Acta Otolaryngol 125: 830-839.

19. Banerjee A, Parnes LS (2005) Intratympanic corticosteroids for sudden idiopathic sensorineural hearing loss. Otol Neurotol 26: 878-881.

20. Westerlaken BO, Stokroos RJ, Dhooge IJ, Wit HP, Albers FW (2003) Treatment of idiopathic sudden sensorineural hearing loss with antiviral therapy: a prospective, randomized, double-blind clinical trial. Ann Otol Rhinol Laryngo 112: $993-1000$

21. Tucci DL, Farmer JC Jr, Kitch RD, Witsell DL (2002) Treatment of sudden sensorineural hearing loss with systemic steroids and valacyclovir. Oto Neurotol 23: 301-308.

22. Fowler KB, Boppana SB (2006) Congenital cytomegalovirus (CMV) infection and hearing deficit. J Clin Virol 35: 226-231.

23. Verbeeck J, Van Kerschaver E, Wollants E, Beuselinck K, Stappaerts L, et al. (2008) Detection of perinatal cytomegalovirus infection and sensorineural hearing loss in belgian infants by measurement of automated auditory brainstem response. J Clin Microbiol 46: 3564-3568.

24. Foulon I, Naessens A, Foulon W, Casteels A, Gordts F (2008) A 10-year prospective study of sensorineural hearing loss in children with congenital cytomegalovirus infection. J Pediatr 153: 84-88.

25. Samileh N, Ahmad S, Mohammad F, Framarz M, Azardokht T, et al. (2008) Role of cytomegalovirus in sensorineural hearing loss of children: a case-control study Tehran, Iran. Int J Pediatr Otorhinolaryngol 72: 203-208.

26. Noorbakhsh S, Memari F, Farhadi M, Tabatabaei A (2008) Sensorineura hearing loss due to Toxoplasma gondii in children: a case-control study. Clin Otolaryngol 33: 269-273.

27. Noorbakhsh S, Farhadi M, Tabatabaei A, Mohamadi S, Jomeh E (2008) Infection in childhood sensory hearing loss. Saudi Med J 29: 1470-1474.

28. Noorbakhsh S, Farhadi M, Tabatabaee A (2006) Comparative study of mumps serology in SNHL children and unaffected ones. J Iran univer Med scien 48 156-164

29. Noorbakhsh S, Siadati A, Farhadi M, Khodapanahandeh F, Monavari H (2006) Sensory hearing loss in children with Mumps infection. Iranian journal of Child hood neurology 2: 125-128 